\title{
ARTICLE
}

Received 12 Apr 2014 | Accepted 10 Jul 2014 | Published 18 Aug 2014 DOl: 10.1038/ncomms5662

\section{Safe storage of radical initiators within a polyaromatic nanocapsule}

Masahiro Yamashina ${ }^{1}$, Yoshihisa Sei ${ }^{1}$, Munetaka Akitaํ \& Michito Yoshizawa ${ }^{1}$

$2,2^{\prime}$-Azobisisobutyronitrile and its derivatives are standard reagents for polymer and organic syntheses that generate radical species on stimuli by light or heat. Radical initiators like the azo compounds are unstable so that they should be kept in the dark at low temperature to avoid photochemical and thermal decomposition as well as accidental explosion. Here we report the spontaneous and quantitative encapsulation of the radical initiators by a supramolecular nanocapsule in aqueous solution. We demonstrate the remarkable stability of the initiators toward light and heat in the well-defined cavity shielded by the polyaromatic capsule shell. The incarcerated and stabilized initiators can be directly utilized for the radical polymerization of olefins on spontaneous release of the initiators from the capsule under the reaction conditions.

\footnotetext{
${ }^{1}$ Chemical Resources Laboratory, Tokyo Institute of Technology, 4259 Nagatsuta, Midori-ku, Yokohama 226-8503, Japan. Correspondence and requests for materials should be addressed to M.Yo. (email: yoshizawa.m.ac@m.titech.ac.jp).
} 
ntrinsic properties of molecules can be altered on encapsulation within artificial or biological nanospaces ${ }^{1-8}$. Stabilization of highly reactive molecules in the confines of molecular capsules enabled the spectroscopic and even crystallographic observation of labile species such as cyclopolyenes ${ }^{9,10}$, benzyne ${ }^{11}$, peroxide $^{12}$, phosphoniums $^{13}$, oligosilanols ${ }^{14}$, iminiums ${ }^{15}$, hemiaminals ${ }^{16}$ and white phosphorus ${ }^{17}$. The capsules function as protective, nanometre-sized containers and shield the guest molecules from external reagents, solvents, water and air. However, there are only a few reports on stabilizing reactive species toward light-triggered reactions through encapsulation ${ }^{18,19}$; the majority of synthetic host frameworks are constructed from aliphatic or small aromatic subunits and are thus unable to shield bound guests from external light. Polyaromatic panels, composed of multiple, extended aromatic rings, effectively absorb ultraviolet (UV) light, with high extinction coefficients and thereby prevent the penetration of photons ${ }^{20,21}$. Therefore, we envisioned that a molecular capsule composed of polyaromatic frameworks could offer a new class of molecular flasks whereby the photo-reactivities of guest substrates can be manipulated through encapsulation.

Here, we report the remarkable stability of radical initiators toward light and heat on encapsulation by supramolecular nanocapsule 1 (Fig. 1a,b). Furthermore, the incarcerated initiators retain their synthetic utility and can initiate the radical polymerization of olefins on release from the capsule, through equilibrium processes, under the reaction conditions. 2,2'Azobisisobutyronitrile (AIBN) and its derivatives (Fig. 1c) are well-known and useful radical initiators for various polymer and organic syntheses on the small scale and are commonly employed in industry at the huge scale ${ }^{22,23}$. Azo-based radical initiators generate organic radical species irreversibly by releasing dinitrogen, triggered by photo or thermal stimuli. The initiators are so unstable due to the thermodynamic favorability of $\mathrm{N}_{2}$ release that they should be stored in the dark at low temperature to prevent photochemical or thermal decomposition and accidental explosion. We reasoned that the stability of photochemically and thermally sensitive radical initiators, AIBN and its derivatives, could be enhanced on encapsulation by capsule 1 by the shielding effect of the polyaromatic frameworks. Supramolecular nanocapsule $\mathbf{1}$ provides a spherical cavity protected by eight anthracene panels (Fig. 1b) ${ }^{24-26}$. The capsule assembles from two $\mathrm{Pd}(\mathrm{II})$ ions and four bent bispyridine ligands 2 with two embedded anthracene rings in a quantitative yield and encapsulates neutral guest molecules with the size up to $1 \mathrm{~nm}$, in which the guests are shaded from the outside by the closed polyaromatic shell.

\section{Results}

Encapsulation of AIBN by capsule 1. Supramolecular capsule 1 spontaneously encapsulated one molecule of AIBN by hydrophobic interactions and the resultant inclusion complex was confirmed by nuclear magnetic resonance (NMR), mass spectrometry (MS) and X-ray crystallographic analyses. When AIBN $(0.75 \mu \mathrm{mol})$ was suspended in a 9:1 $\mathrm{D}_{2} \mathrm{O} / \mathrm{CD}_{3} \mathrm{CN}$ solution $(0.5 \mathrm{ml})$ of capsule $1(0.75 \mu \mathrm{mol})$ at room temperature, the $1: 1$ inclusion complex, $1 \supset A I B N$, was formed quantitatively within $1 \mathrm{~min}$ (Fig. 2a). In the ${ }^{1} \mathrm{H}$ NMR spectrum, the methyl signal derived from AIBN was desymmetrized and observed at -1.95 and -1.81 p.p.m. with outstanding upfield shifts $\left(\Delta \delta_{\max }=-3.65\right.$ p.p.m.) due to the encapsulation in the capsule with tightly wound anthracene panels (Fig. 2b,c). A slight shift of the inner phenylene signal $\left(H_{\mathrm{a}}\right)$ also indicated the accommodation of AIBN in 1. Quantitative formation of a 1:1 host-guest complex was demonstrated by ${ }^{1} \mathrm{H}$ NMR integration and electrospray ionization time-of-flight (ESI-TOF) MS analysis: prominent MS signals observed at $\mathrm{m} / \mathrm{z} 900.7,1,222.2$ and $1,863.8$ were assigned to $\left[1 \supset \mathrm{AIBN}_{-} n \bullet \mathrm{NO}_{3}^{-}\right]^{n+}$ species $(n=4,3$ and 2, respectively; Fig. 2d). Finally, pale-yellow single crystals were obtained by slow concentration of a $\mathrm{H}_{2} \mathrm{O} / \mathrm{CH}_{3} \mathrm{CN}(9: 1)$ solution of $1 \supset$ AIBN over 1 week. Crystallographic analysis of $1 \supset$ AIBN confirmed that the single AIBN guest molecule is shielded from the external environment by the anthracene panels of capsule 1 (Fig. 2e,f). The crystal structure also revealed that the enclathrated AIBN guest adopts an S-shaped trans configuration, where the $\mathrm{CN}$ groups point toward the $\mathrm{Pd}$ centres (where the CN-Pd distances are $\sim 0.35 \mathrm{~nm}$ ) in the cavity. Infrared (IR) spectroscopy suggested the existence of weak electrostatic interactions between the $\mathrm{CN}$ groups and the $\mathrm{Pd}$ ions.

Photostability of AIBN and its derivatives within capsule 1 . The AIBN initiator was found to be stable toward UV light in the confines of capsule 1 . A solution of $1 \supset \mathrm{AIBN}$ in $\mathrm{D}_{2} \mathrm{O} / \mathrm{CD}_{3} \mathrm{CN}$ $(1.5 \mathrm{mM})$ was irradiated for $10 \mathrm{~h}$ at room temperature, whereon ${ }^{1} \mathrm{H}$ NMR analysis revealed the AIBN molecule encapsulated within the capsule remained intact (Fig. 3a,b). Free AIBN, on the other hand, completely decomposed into tetramethylsuccinonitrile (TMSN) and other by-products under similar conditions $\left(\lambda_{\text {irrad }}=360 \mathrm{~nm}\right.$ for $10 \mathrm{~h}$ in $\left.\mathrm{CD}_{3} \mathrm{OD}\right)$ in the absence of the capsule (Fig. 3c,d). Kinetic analysis of the decomposition revealed half-lives $\left(\tau_{1 / 2}\right)$ of $\geq 690$ and $1.8 \mathrm{~h}$ for the encapsulated AIBN and free AIBN, respectively (Fig. 3e). Thus AIBN is stabilized toward UV light by $\geq 380$ times within the capsule (See Supplementary Table 1). We attribute the observed stability of enclathrated AIBN

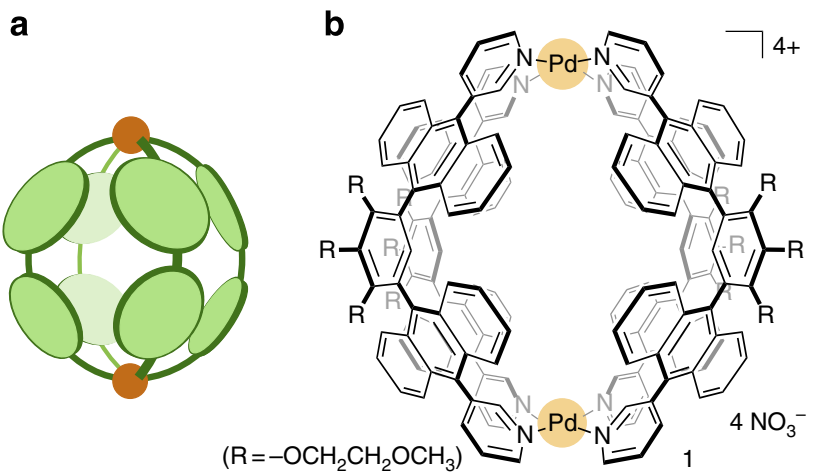

C<smiles>[R]C(C)(C#N)N=NC([R])(C)C#N</smiles>

AIBN $\left(\mathrm{R}=-\mathrm{CH}_{3}\right)$

AMBN $\left(\mathrm{R}=-\mathrm{CH}_{2} \mathrm{CH}_{3}\right)$

AMMVN $\left(\mathrm{R}=-\mathrm{CH}_{2} \mathrm{C}\left(\mathrm{CH}_{3}\right)_{2} \mathrm{OCH}_{3}\right)$

Figure 1 | Structure of a supramolecular nanocapsule and radical initiators. (a) Cartoon and (b) chemical structure of nanocapsule 1 and (c) radical initiators: AIBN, AMBN and AMMVN. 
a

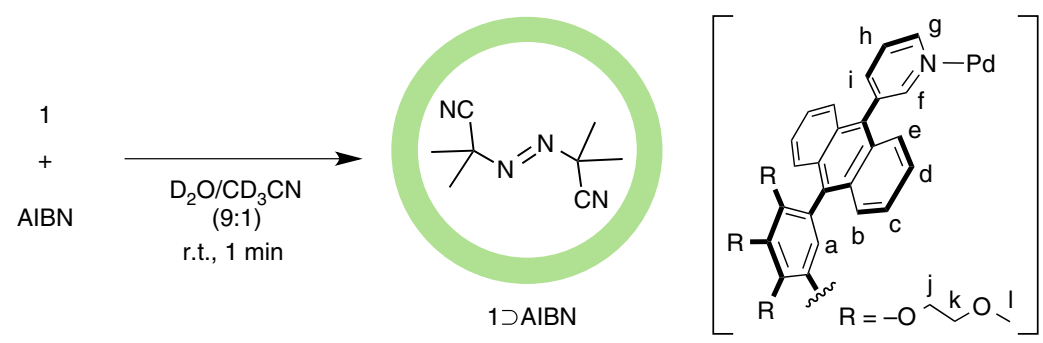

b

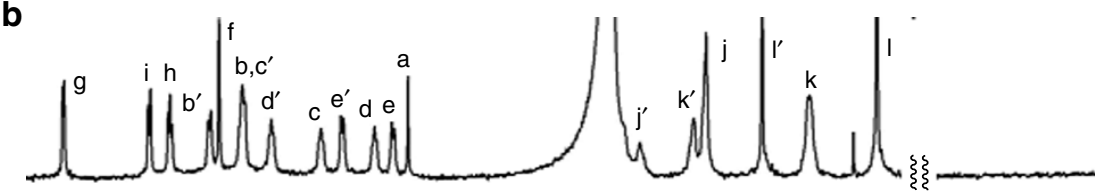

c

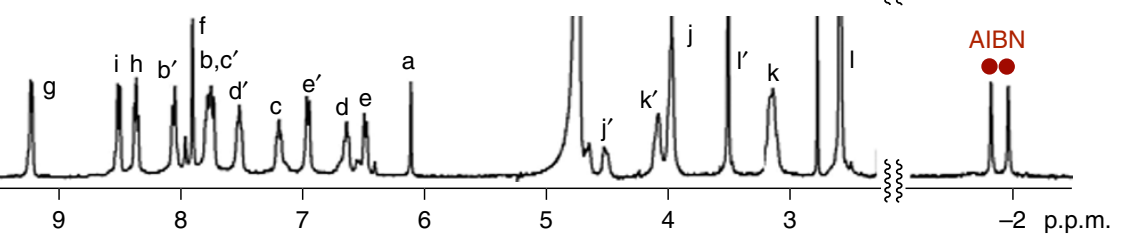

d

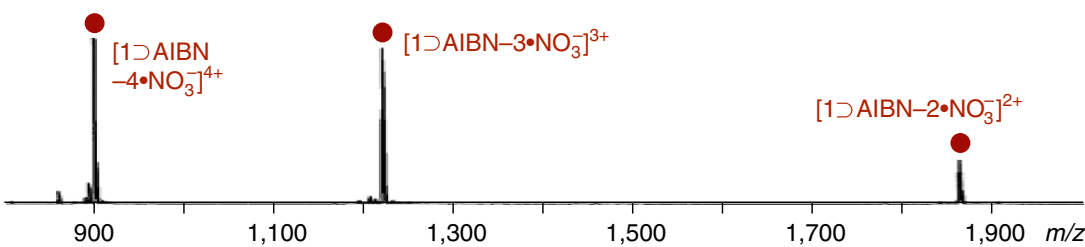

e

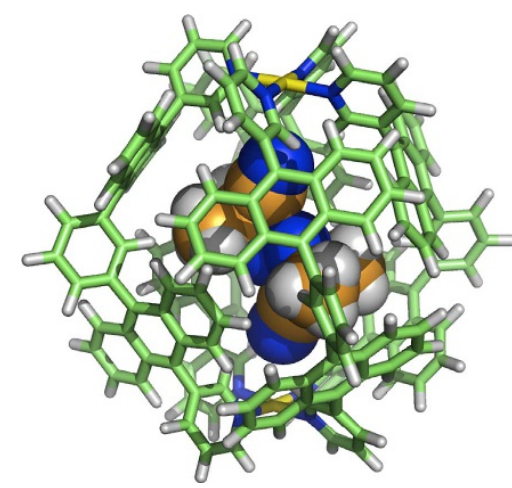

f

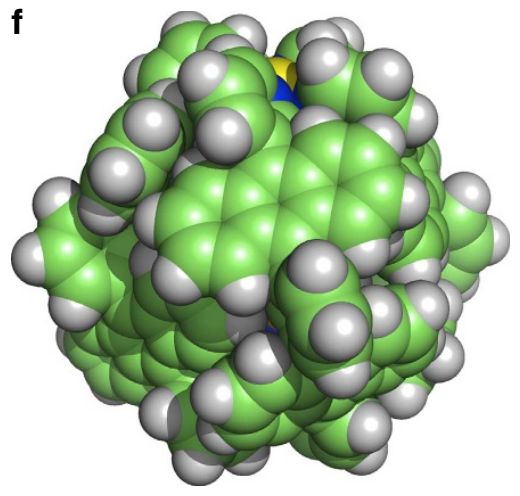

Figure 2 | Formation and characterization of capsule $1 \supset$ AIBN. (a) Schematic representation of the encapsulation of AIBN within capsule 1 in aqueous solution. ${ }^{1} \mathrm{H}$ NMR spectra $\left(400 \mathrm{MHz}\right.$, room temperature, $\mathrm{D}_{2} \mathrm{O} / \mathrm{CD}_{3} \mathrm{CN}(9 / 1)$ ) of (b) capsule $\mathbf{1}$ and (c) $1 \supset$ AIBN. (d) ESI-TOF MS spectrum of $\mathbf{1} \supset \mathrm{AIBN}$. X-ray crystal structure of $\mathbf{1} \supset \mathrm{AIBN}$ : (e) space-filling (for AIBN) and cylindrical (for $\mathbf{1})$ representation and (f) space-filling representation (substituents and counterions are omitted for clarity).

toward light to the optical shielding of the polyaromatic shell of capsule 1 (Fig. 2f). UV-visible spectrum of capsule 1 showed strong absorption bands $\left(\varepsilon=72,0001 \mathrm{~mol}^{-1} \mathrm{~cm}^{-1}\right.$ at $\left.380 \mathrm{~nm}\right)$ in the range of $300-450 \mathrm{~nm}$ due to the $\pi-\pi^{*}$ transitions of the embedded anthracene moieties. The bands fully occlude the weak absorption band of AIBN $\left(\varepsilon=141 \mathrm{~mol}^{-1} \mathrm{~cm}^{-1}\right.$ at $\left.345 \mathrm{~nm}\right)$, illustrating that the encapsulated initiators are effectively shielded from UV light in the capsule.

Similar to AIBN, the slightly larger radical initiator, 2,2'-azobis(2-methylbutyronitrile) (AMBN), was quantitatively encapsulated in capsule 1 and the resultant host-guest complex also displayed stability for UV light irradiation (See Supplementary Table 1). The $\tau_{1 / 2}$ for the decomposition of AMBN within 1 was estimated to be over $690 \mathrm{~h}$, indicating that the photostability is more than 580 times enhanced as compared with that of free AMBN (Fig. 3e). The even larger AIBN derivative, 2,2'-azobis(4-methoxy-2,4-dimethylvaleronitrile)
$(\mathrm{AMMVN})^{27}$ was also encapsulated by capsule $\mathbf{1}$. Although the diameter of the radical initiator $(1.6 \mathrm{~nm})$ is longer than that of the inner cavity of $\mathbf{1}(\sim 1.0 \mathrm{~nm})$, a single molecule of AMMVN entered the cavity of $\mathbf{1}$ in $\mathrm{H}_{2} \mathrm{O} / \mathrm{CH}_{3} \mathrm{CN}$ solution, as confirmed by NMR and MS analyses (Fig. 4a-c). Host-guest complex $1 \supset A M M V N$ showed high stability against UV light: $\tau_{1 / 2}$ values for the decomposition of AMMVN within and without 1 were 70 and $1.5 \mathrm{~h}$, respectively (Fig. $4 \mathrm{~d}$ ). These results further demonstrate that capsule $\mathbf{1}$ can serves as a molecular container for safekeeping radical initiators under light.

Thermal stability of AMMVN within capsule 1 . Remarkably, in the polyaromatic shell of capsule 1, AMMVN remained intact at room temperature for several weeks and on heating at $50^{\circ} \mathrm{C}$ for over $10 \mathrm{~h}$ (Fig. 4d). AMMVN is one of the most thermally reactive initiators among reported AIBN derivatives and decomposition 

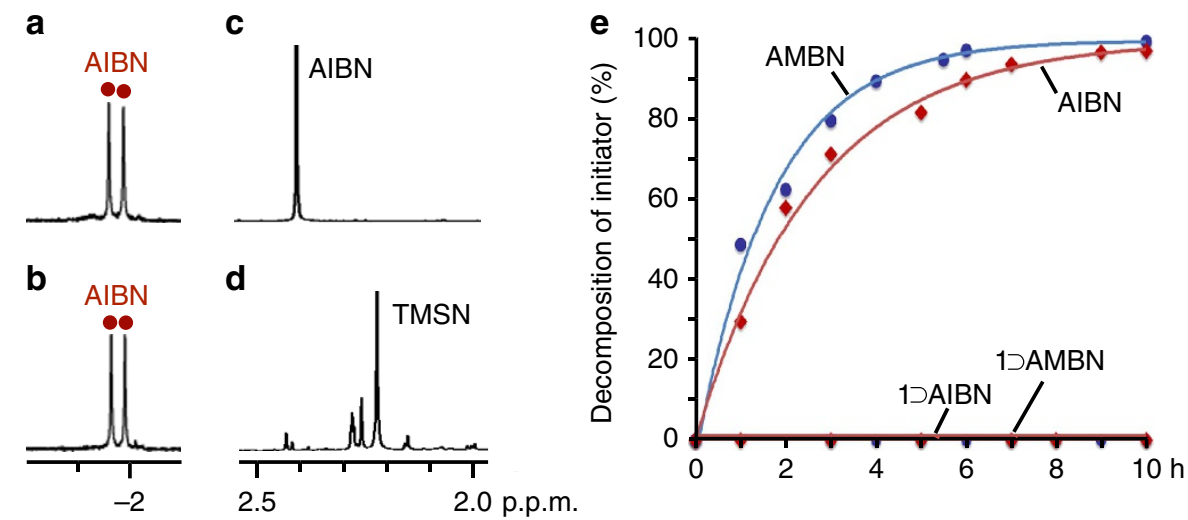

Figure 3 | Photostability of radical initiators AIBN and AMBN within/without capsule 1. ${ }^{1} \mathrm{H}$ NMR spectra ( $400 \mathrm{MHz}$, room temperature) of $1 \supset \mathrm{AIBN}$ (a) before and (b) after UV irradiation $\left(\lambda_{\text {irrad }}=360 \mathrm{~nm}\right.$ ) in $\mathrm{D}_{2} \mathrm{O} / \mathrm{CD}_{3} \mathrm{CN}(9 / 1)$ for $10 \mathrm{~h}$ and free AIBN (c) before and (d) after UV light irradiation $\left(\lambda_{\text {irrad }}=360 \mathrm{~nm}\right)$ in $\mathrm{CD}_{3} \mathrm{OD}$ for $10 \mathrm{~h}$. (e) Time course of the decomposition of AIBN and AMBN within/without $\mathbf{1}$ on UV irradiation $\left(\lambda_{\text {irrad }}=360 \mathrm{~nm}\right)$ at room temperature.

a

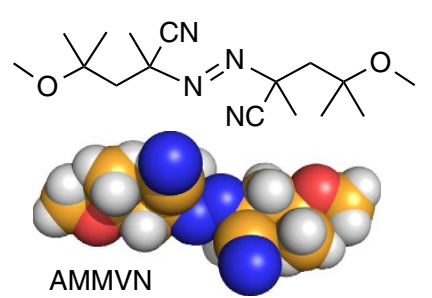

b

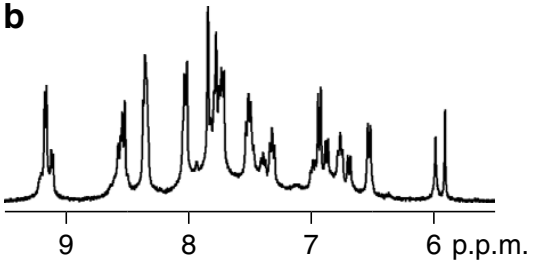

C

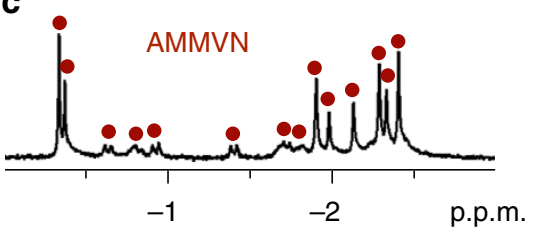

e

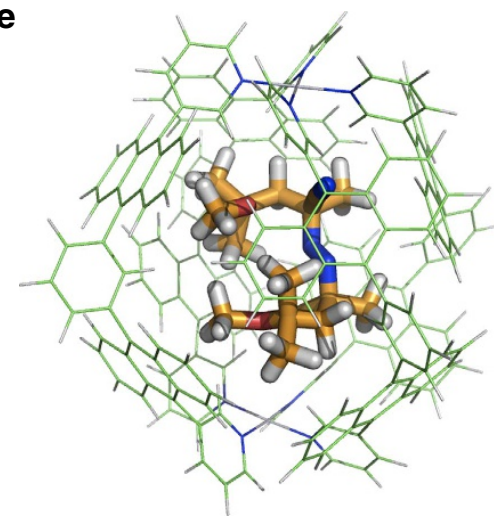

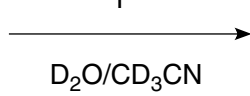

r.t.

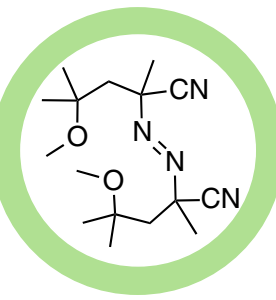

$1 \supset$ AMMVN

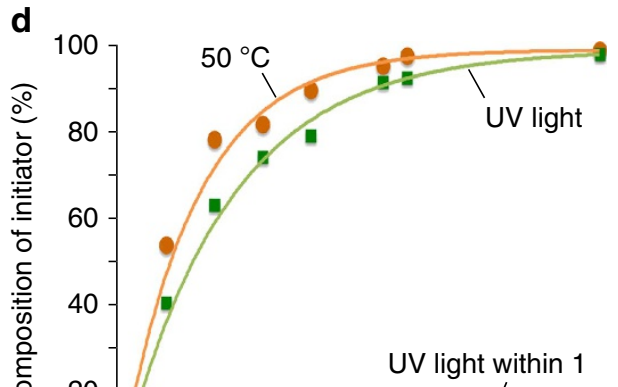

UV light within 1

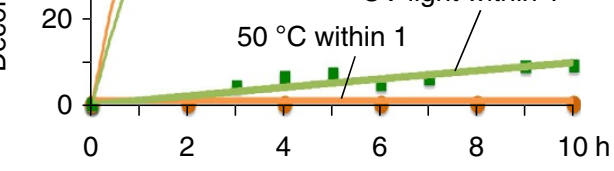

f

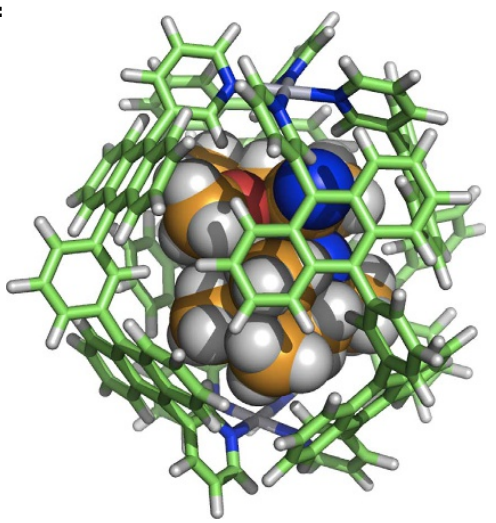

Figure 4 | Encapsulation, characterization and stabilization of AMMVN within capsule 1. (a) Schematic representation of the encapsulation of $A M M V N$ within capsule $\mathbf{1}$ in aqueous solution. ${ }^{1} \mathrm{H}$ NMR spectra $\left(400 \mathrm{MHz}\right.$, room temperature, $\mathrm{D}_{2} \mathrm{O} / \mathrm{CD}_{3} \mathrm{CN}(9 / 1)$ ) of $\mathbf{1} \supset \mathrm{AMMVN}$ in (b) the aromatic and (c) aliphatic regions. (d) Photostability $\left(\lambda_{\text {irrad }}=360 \mathrm{~nm}\right.$, room temperature) and thermal stability $\left(50^{\circ} \mathrm{C}\right.$, dark) of $A M M V N$ within/without 1 . X-ray crystal structure of $\mathbf{1}^{\prime} \mathrm{AMMVN}$ : (e) stick (for $\mathbf{1}^{\prime}$ ) and cylindrical (for AMMVN) and (f) cylindrical (for $\mathbf{1}^{\prime}$ ) and space-filling (for AMMVN) representations (substituents and counterions are omitted for clarity). 
occurs even at room temperature (for example, $\tau_{1 / 2}=10 \mathrm{~h}$ at $30^{\circ} \mathrm{C}$ and $\tau_{1 / 2}=1.1 \mathrm{~h}$ at $50^{\circ} \mathrm{C}$; Fig. $4 \mathrm{~d}$ ). In the solid state, AMMVN completely decomposed at $50^{\circ} \mathrm{C}$ (or under UV irradiation $\left(\lambda_{\max }=360 \mathrm{~nm}\right)$ ) for $10 \mathrm{~h}$, whereas AMMVN encapsulated by the capsule remained intact under the same conditions. This unusual thermal stability can be rationalized through the tight encapsulation of the large radical initiator by capsule $\mathbf{1}$ through hydrophobic interactions. Elongation of the $\mathrm{N}-\mathrm{C}$ bonds $(\Delta d=0.05 \mathrm{~nm})$ of AIBN and the derivatives along the reaction pathway is essential for the generation of the radical species through homolytic bond cleavage ${ }^{28}$. However, AMMVN fully fills the capsule cavity and thereby the formation of the lengthened transition state, with accompanying bond lengthening and volume expansion, should be energetically disfavored. X-ray crystallographic analysis of 1' $\supset$ AMMVN (which is an analogue of $1 \supset A M M V N$, where the $\mathrm{Pd}(\mathrm{II})$ ions are replaced by $\mathrm{Pt}(\mathrm{II})$ ions) revealed that the AMMVN guest occupies $64 \%$ of the cavity volume $\left(\sim 0.55 \mathrm{~nm}^{3}\right)$ by using VOIDOO program (See Supplementary Table 2$)^{29}$. In the crystal structure, the encapsulated AMMVN adopts an otherwise labile C-shaped conformation arising from structural complementarity with the spherical cavity (Fig. 4e,f) ${ }^{30-32}$. Gas-phase calculations (DFT, $\mathrm{B} 3 \mathrm{LYP} / 6-31 \mathrm{G}^{*}$ level) indicated the energy of the observed conformation of AMMVN is $>60 \mathrm{kcal} \mathrm{mol}^{-1}$ higher than that of the optimized conformation without the capsule (See Supplementary Table 3). We hypothesize that a tight fit is essential for thermal stabilization. Host-guest complexes of the smaller AIBN and AMBN with 1 showed no increase in thermal stability relative to the free guests in organic solution: AIBN and AMBN completely decomposed at $65^{\circ} \mathrm{C}$ for $48 \mathrm{~h}$ in free solution and even within host $\mathbf{1}$. Interestingly, the thermal decomposition of AIBN within 1 gave rise to TMSN in quantitative yield, whereas that without 1 yielded a mixture of TMSN ( 20\%) and other by-products $(\sim 80 \%)$ under similar conditions.

Thermal and photochemical polymerization of MMA. Encapsulated $\mathbf{1} \supset \mathrm{AIBN}$ and $\mathbf{1} \supset \mathrm{AMMVN}$ retained reactivity as radical initiators for standard radical polymerization reactions in organic solution through release of the initiators from the capsule (Fig. 5). The initiators are bound in the capsule by non-covalent hydrophobic interactions and thus subject to reversible binding processes under equilibrium. A pale-yellow powder of $1 \supset$ AIBN was obtained from the aqueous solution on solvent evaporation.
A toluene solution $(100 \mu \mathrm{l})$ of methyl methacrylate (MMA; $1.9 \mathrm{mmol})$ and $\mathbf{1} \supset \mathrm{AIBN}(0.75 \mu \mathrm{mol},[\mathrm{MMA}] /[\mathbf{1} \supset \mathrm{AIBN}]=2,533)$ was then heated at $80^{\circ} \mathrm{C}$ for $24 \mathrm{~h}$ and the resultant solution solidified due to poly(methylmethacrylate) (PMMA) formation. The polymer was isolated by the addition of a $\mathrm{CH}_{2} \mathrm{Cl}_{2}$ solution of the reaction mixtures into $\mathrm{CH}_{3} \mathrm{OH}$ and was washed with $\mathrm{CH}_{3} \mathrm{OH}$. The yield and average molecular weight $\left(M_{\mathrm{n}}\right)$ of the obtained PMMA were determined to be $84 \%$ and $1.08 \times 10^{5}$, respectively. These results are comparable to PMMAs prepared by the use of free AIBN $(0.75 \mu \mathrm{mol})$ under similar conditions (See Supplementary Table 4$)$. The molecular weight distribution of the obtained polymers $\left(M_{\mathrm{w}} / M_{\mathrm{n}}=2.8\right)$ was insensitive to the existence of the capsule. Photopolymerization of MMA in toluene solution containing $1 \supset$ AIBN gave rise to similar PMMA (86\% yield, $\left.M_{\mathrm{w}}=1.61 \times 10^{5}\right)$ on irradiation by a Xe lamp $(150 \mathrm{~W})$ for $24 \mathrm{~h}$ at room temperature. The $1 \supset A M M V N$ initiator complex also successfully initiated the thermal and photochemical polymerizations of MMA (See Supplementary Table 4).

\section{Discussion}

In this study, we successfully controlled the stabilities of wellknown and widely used radical initiators, AIBN and its derivatives, toward light through encapsulation within a supramolecular nanocapsule with polyaromatic frameworks. The encapsulation process occurs quantitatively by hydrophobic host-guest interactions in less than one minute in aqueous solution at room temperature. The encapsulated initiators in the capsule were significantly stabilized against light irradiation (up to 580 times) due to optical filtering by the polyaromatic frameworks. Furthermore, the thermal stabilization of large AIBN derivative (645 times) was accomplished through encapsulation in tight fitting molecular capsule of complementary size and shape. The safely stored initiators could be used for usual olefin polymerization in organic solvent through spontaneous release of the initiators. We emphasize that well-defined, constrictive nanospaces enclosed by a self-assembled polyaromatic shell are ideal for manipulating both the photochemical and thermal reactivity of encapsulated guest molecules.

\section{Methods}

General. NMR: Bruker AVANCE-400 (400 MHz), ESI-TOF MS: Bruker micrOTOF II, FT-IR: JASCO FT/IR-4200, UV-vis: JASCO V-670DS, UV light irradiation: Soma Kogaku Xe lamp $(150 \mathrm{~W})$ at a distance of $7.0 \mathrm{~cm}$, X-ray: APEXII DUO/CCD diffractometer, GPC: TOSO HLC-8120GPC $\left(40^{\circ} \mathrm{C}, 0.5 \mathrm{ml} \mathrm{min}^{-1}\right)$
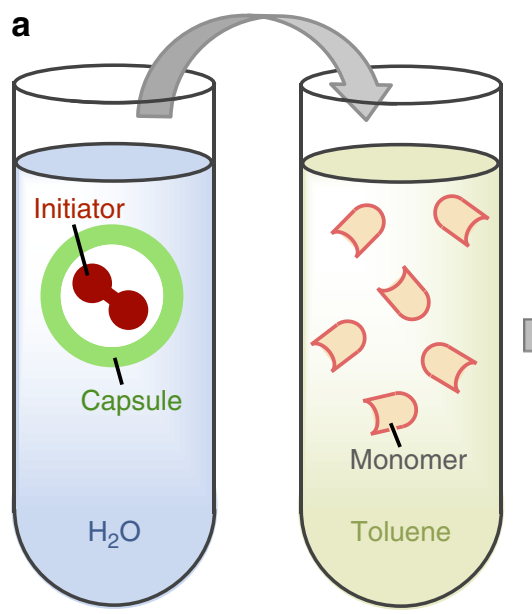

b

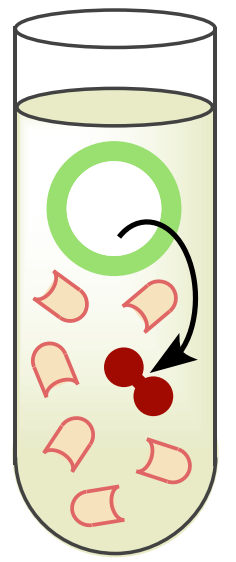

C

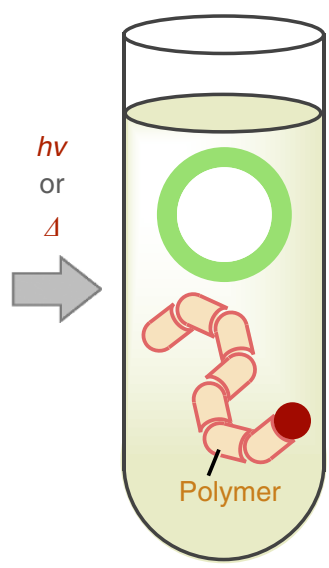

Figure 5 | Schematic representation of olefin polymerization with capsule 1 containing a radial initiator. (a) Addition of the capsulated initiator to an organic solution of monomers. (b) Spontaneous release of the initiator from the capsule. (c) Polymerization of the monomers initiated by the initiator on photo or thermal stimuli. 
with Shodex SB-802.5 HQ and SB-806 M HQ columns. Solvents and reagents: TCI Co., Ltd., WAKO Pure Chemical Industries Ltd., KANTO kagaku KANTO CHEMICAL CO., INC., Sigma-Aldrich Co. and Cambridge Isotope Laboratories, Inc. Ligand $\mathbf{2}$ and Pd-capsule 1 (See Supplementary Methods and Supplementary Figs 1-3) were synthesized according to previously reported procedures ${ }^{24,33}$.

Formation of $1 \supset$ AlBN. Pd-capsule $\mathbf{1}(2.8 \mathrm{mg}, 0.75 \mu \mathrm{mol})$, AIBN $(0.12 \mathrm{mg}$, $0.75 \mu \mathrm{mol}), \mathrm{D}_{2} \mathrm{O}(0.45 \mathrm{ml})$ and $\mathrm{CD}_{3} \mathrm{CN}(0.05 \mathrm{ml})$ were added to a brown glass test tube. The mixture was stirred at room temperature for $1 \mathrm{~min}$. The quantitative formation of a $1 \supset$ AIBN complex was confirmed by NMR and ESI-TOF MS analyses (See Supplementary Figs 4-7). Pale-yellow single crystals suitable for $\mathrm{X}$-ray crystallographic analysis were obtained by slow concentration of a $\mathrm{H}_{2} \mathrm{O}(+\mathrm{NaCl}) / \mathrm{CH}_{3} \mathrm{CN}(9: 1)$ solution of $\mathbf{1} \supset \mathrm{AIBN}$ at room temperature over 1 week (See Supplementary Fig. 8 and Supplementary Table 5). Similarly, a $1 \supset$ AMBN complex was prepared in quantitative yield (See Supplementary Methods and Supplementary Figs 9-12).

${ }^{1} \mathrm{H}$ NMR $\left(400 \mathrm{MHz}, \mathrm{D}_{2} \mathrm{O}: \mathrm{CD}_{3} \mathrm{CN}=9: 1\right.$, room temperature): $\delta-1.95(\mathrm{~s}, 6 \mathrm{H}$, AIBN), -1.81 (s, 6H, AIBN), $2.56(\mathrm{~s}, 24 \mathrm{H}), 3.21(\mathrm{~m}, 16 \mathrm{H}), 3.49(\mathrm{~s}, 12 \mathrm{H}), 3.84-4.15$ (m, $24 \mathrm{H}), 4.41-4.67(\mathrm{~m}, 8 \mathrm{H}), 6.08(\mathrm{~s}, 4 \mathrm{H}), 6.46(\mathrm{~d}, J=8.4 \mathrm{~Hz}, 8 \mathrm{H}), 6.61(\mathrm{dd}, J=7.2$, $8.4 \mathrm{~Hz}, 8 \mathrm{H}), 6.93(\mathrm{~d}, J=8.4 \mathrm{~Hz}, 8 \mathrm{H}), 7.17(\mathrm{dd}, J=7.2,8.4 \mathrm{~Hz}, 8 \mathrm{H}), 7.51(\mathrm{dd}, J=7.2$, $8.4 \mathrm{~Hz}, 8 \mathrm{H}), 7.72-7.77(\mathrm{~m}, 16 \mathrm{H}), 7.89(\mathrm{~s}, 8 \mathrm{H}), 8.04(\mathrm{~d}, J=8.4 \mathrm{~Hz}, 8 \mathrm{H}), 8.35(\mathrm{dd}$, $J=5.6,7.6 \mathrm{~Hz}, 8 \mathrm{H}), 8.49(\mathrm{~d}, J=7.6 \mathrm{~Hz}, 8 \mathrm{H}), 9.20(\mathrm{~d}, J=5.6 \mathrm{~Hz}, 8 \mathrm{H}) . \mathrm{IR}(\mathrm{KBr}$, $\mathrm{cm}^{-1}$ ): $3,062,2,983,2,929,2,882,2,817,2,239,1,945,1,637,1,384,1,194,1,125$, $1,104,1,065,1,029,943,768,706,671,644$. ESI-TOF MS $\left(\mathrm{H}_{2} \mathrm{O}\right): \mathrm{m} / z 1,863.8$ $\left[\mathbf{1} \supset \mathrm{AIBN}-2 \mathrm{NO}_{3}^{-}\right]^{2+}, 1,222.2\left[1 \supset \mathrm{AIBN}-3 \mathrm{NO}_{3}^{-}\right]^{3+}, 900.7[\mathbf{1} \supset \mathrm{AIBN}-$ $\left.4 \mathrm{NO}_{3}^{-}\right]^{4+}$.

Formation of $1 \supset$ AMMVN. Pd-capsule $1(2.8 \mathrm{mg}, 0.75 \mu \mathrm{mol})$, AMMVN $(0.23 \mathrm{mg}$, $0.75 \mu \mathrm{mol}), \mathrm{D}_{2} \mathrm{O}(0.45 \mathrm{ml})$ and $\mathrm{CD}_{3} \mathrm{CN}(0.05 \mathrm{ml})$ were added to a brown glass test tube. The mixture was stirred at room temperature for $1 \mathrm{~min}$. The quantitative formation of a $1 \supset$ AMMVN complex was confirmed by NMR and ESI-TOF MS analyses (See Supplementary Figs 13-16). Pale-yellow single crystals suitable for X-ray crystallographic analysis were obtained by slow concentration of a $\mathrm{H}_{2} \mathrm{O}$ / $\mathrm{CH}_{3} \mathrm{CN}$ (9:1) solution of $\mathbf{1}^{\prime} \supset \mathrm{AMMVN}$ at room temperature over 1 week (See Supplementary Methods and Supplementary Figs 17-21 and Supplementary Table 6).

${ }^{1} \mathrm{H}$ NMR $\left(400 \mathrm{MHz}, \mathrm{D}_{2} \mathrm{O}: \mathrm{CD}_{3} \mathrm{CN}=9: 1\right.$, room temperature): $\delta-2.39(\mathrm{~s}$, AMMVN), -2.31 (s, AMMVN), -2.27 (s, AMMVN), -2.11 (s, AMMVN), -1.97 (s, AMMVN), -1.89 (s, AMMVN), -1.70 (br, AMMVN), -1.38 (d, $J=14.0 \mathrm{~Hz}$, AMMVN), $-0.90(\mathrm{~d}, J=14.0 \mathrm{~Hz}$, AMMVN $),-0.80(\mathrm{~d}, J=14.0 \mathrm{~Hz}$, AMMVN), -0.61 (d, $J=14.0 \mathrm{~Hz}$, AMMVN), -0.35 (s, AMMVN), -0.31 (s, AMMVN), 2.61-2.65 (m, 24H), 3.02-3.32 (m, 16H), 3.54 (d-like, 12H), $3.86-4.28(\mathrm{~m}, 24 \mathrm{H}), 4.28-4.69(\mathrm{~m}, 8 \mathrm{H}), 5.94-6.02(\mathrm{~m}, 4 \mathrm{H}), 6.56(\mathrm{~d}, J=8.8 \mathrm{~Hz}, 4 \mathrm{H})$, 6.71-7.02 (m, 20H), 7.33-7.50 (m, 8H), $7.54(\mathrm{dd}, J=7.2,8.8 \mathrm{~Hz}, 8 \mathrm{H}), 7.74-7.81$ $(\mathrm{m}, 16 \mathrm{H}), 7.87(\mathrm{~s}, 8 \mathrm{H}), 8.05(\mathrm{~d}, J=8.8 \mathrm{~Hz}, 8 \mathrm{H}), 8.39(\mathrm{br}, 8 \mathrm{H}), 8.56-8.61(\mathrm{~m}, 8 \mathrm{H})$, 9.14-9.21 (m, 8H). IR (KBr, cm $\left.{ }^{-1}\right): 3,063,2,978,2,933,2,883,2,822,2,239,1,950$, $1,839,1,655,1,384,1,242,1,195,1,065,1,029,942,769,706,670,611$. ESI-TOF MS $\left(\mathrm{H}_{2} \mathrm{O}\right): m / z$ 1,936.6 [1 $\supset$ AMMVN-2NO $\left.{ }_{3}^{-}\right]^{2+}, 1,270.4[1 \supset$ AMMVN$\left.3 \mathrm{NO}_{3}^{-}\right]^{3+}, 937.1\left[\mathbf{1} \supset \mathrm{AMMVN}-4 \mathrm{NO}_{3}^{-}\right]^{4+}$.

Photostability of AIBN within 1. $1 \supset$ AIBN complex $(2.9 \mathrm{mg}, 0.75 \mu \mathrm{mol}), \mathrm{D}_{2} \mathrm{O}$ $(0.45 \mathrm{ml})$ and $\mathrm{CD}_{3} \mathrm{CN}(0.05 \mathrm{ml})$ were added to a NMR tube under $\mathrm{N}_{2} .{ }^{1} \mathrm{H}$ NMR spectrum of the solution was measured before and after UV light irradiation $(360 \mathrm{~nm})$ at room temperature for $10 \mathrm{~h}$ (See Supplementary Figs 22-24). Similarly, the photostability of AMBN within 1 was studied (See Supplementary Figs 24-26).

Photostability of AMMVN within 1. $1 \supset$ AMMVN complex (3.0 mg, $0.75 \mu \mathrm{mol})$, $\mathrm{D}_{2} \mathrm{O}(0.45 \mathrm{ml})$ and $\mathrm{CD}_{3} \mathrm{CN}(0.05 \mathrm{ml})$ were added to a NMR tube under $\mathrm{N}_{2} .{ }^{1} \mathrm{H}$ NMR spectrum of the solution was measured before and after UV light irradiation $(360 \mathrm{~nm})$ at room temperature for $10 \mathrm{~h}$ (See Supplementary Figs 24 and 27-29). Photostability of AMMVN within $\mathbf{1}$ in the solid state was also studied (See Supplementary Fig. 30).

Thermal stability of AIBN within 1. $1 \supset$ AIBN complex $(2.9 \mathrm{mg}, 0.75 \mu \mathrm{mol}), \mathrm{D}_{2} \mathrm{O}$ $(0.45 \mathrm{ml})$ and $\mathrm{CD}_{3} \mathrm{CN}(0.05 \mathrm{ml})$ were added to a NMR tube. ${ }^{1} \mathrm{H}$ NMR spectrum of the solution was measured before and after heating at $65^{\circ} \mathrm{C}$ for 2 days (See Supplementary Figs 24 and 31). Similarly, the thermal stability of AMBN within 1 was studied (See Supplementary Methods and Supplementary Figs 24 and 32).

Thermal stability of AMMVN within 1. $1 \supset A M M V N$ complex $(3.0 \mathrm{mg}$, $0.75 \mu \mathrm{mol}), \mathrm{D}_{2} \mathrm{O}(0.45 \mathrm{ml})$ and $\mathrm{CD}_{3} \mathrm{CN}(0.05 \mathrm{ml})$ were added to a NMR tube. ${ }^{1} \mathrm{H}$ NMR spectrum of the solution was measured before and after heating at $50{ }^{\circ} \mathrm{C}$ for $33 \mathrm{~h}$ (See Supplementary Figs 24, 33 and 34). Thermal stability of AMMVN within $\mathbf{1}$ in the solid state was also studied (See Supplementary Fig. 35).

Thermal polymerization of MMA by AIBN or $1 \supset$ AIBN. AIBN $(0.12 \mathrm{mg}$, $0.75 \mu \mathrm{mol})$ or $1 \supset \mathrm{AIBN}(2.9 \mathrm{mg}, 0.75 \mu \mathrm{mol})$, MMA $(0.2 \mathrm{ml}, 1.9 \mathrm{mmol})$ and toluene
$(0.1 \mathrm{ml})$ were added to a $5 \mathrm{ml}$ Schlenk flask under $\mathrm{N}_{2}$. The mixture was heated at $80^{\circ} \mathrm{C}$ for $24 \mathrm{~h}$. The crude product was dissolved to $\mathrm{CH}_{2} \mathrm{Cl}_{2}(5 \mathrm{ml})$ and then the resultant solution was poured into methanol $(50 \mathrm{ml})$. The precipitate was collected, washed with methanol and dried under reduced pressure to afford PMMA as a white solid (166 mg (88\% yield) from AIBN initiator, $159 \mathrm{mg}(84 \%$ yield) from $1 \supset$ AIBN initiator; See Supplementary Figs 36 and 37 and Supplementary Table 4). Thermal polymerization of MMA by $1 \supset$ AMMVN also afforded PMMA (See Supplementary Methods and Supplementary Fig. 38 and Supplementary Table 4).

Photopolymerization of MMA by AIBN or $1 \supset$ AIBN. AIBN $(0.12 \mathrm{mg}, 0.75 \mu \mathrm{mol})$ or $\mathbf{1} \supset$ AIBN $(2.9 \mathrm{mg}, 0.75 \mu \mathrm{mol})$, MMA $(0.2 \mathrm{ml}, 1.9 \mathrm{mmol})$ and toluene $(0.05 \mathrm{ml})$ were added to a $5 \mathrm{ml}$ Schlenk flask under $\mathrm{N}_{2}$. The mixture was irradiated with a UV lamp (no cut filter) at room temperature for $24 \mathrm{~h}$. The crude product was dissolved to $\mathrm{CH}_{2} \mathrm{Cl}_{2}(5 \mathrm{ml})$ and then the resultant solution was poured into methanol $(50 \mathrm{ml})$ The precipitate was collected, washed with methanol and dried under reduced pressure to afford PMMA as a white solid (164 $\mathrm{mg}$ (87\% yield) from AIBN initiator, $162 \mathrm{mg}$ (86\% yield) from $1 \supset$ AIBN initiator; See Supplementary Fig. 39 and Supplementary Table 4). Photopolymerization of MMA by $1 \supset$ AMMVN also afforded PMMA (See Supplementary Methods and Supplementary Fig. 40 and Supplementary Table 4).

\section{References}

1. Hof, F., Craig, S. L., Nuckolls, C. \& Rebek, Jr J. Molecular encapsulation. Angew. Chem. Int. Ed. 41, 1488-1508 (2002).

2. Vriezema, D. M. et al. Self-assembled nanoreactors. Chem. Rev. 105, 1445-1489 (2005).

3. Yoshizawa, M., Klosterman, J. K. \& Fujita, M. Functional molecular flasks: new properties and reactions within discrete, self-assembled hosts. Angew. Chem. Int. Ed. 48, 3418-3438 (2009).

4. Amouri, H., Desmarets, C. \& Moussa, J. Confined nanospaces in metallocages: guest molecules, weakly encapsulated anions, and catalyst sequestration. Chem. Rev. 112, 2015-2041 (2012).

5. Cook, T. R., Zheng, Y.-R. \& Stang, P. J. Metal-organic frameworks and selfassembled supramolecular coordination complexes: comparing and contrasting the design, synthesis, and functionality of metal-organic materials. Chem. Rev. 113, 734-777 (2013)

6. Smulders, M. M. J., Riddell, I. A., Browne, C. \& Nitschke, J. R. Building on architectural principles for three-dimensional metallosupramolecular construction. Chem. Soc. Rev. 42, 1728-1754 (2013).

7. Pluth, M. D., Bergman, R. G. \& Raymond, K. N. Acid catalysis in basic solution a supramolecular host promotes orthoformate hydrolysis. Science 316, 85-88 (2007).

8. Kaanumalle, L. S., Gibb, C. L. D., Gibb, B. C. \& Ramamurthy, V. Controlling photochemistry with distinct hydrophobic nano-environments. J. Am. Chem. Soc. 126, 14366-14367 (2004).

9. Cram, D. J., Tanner, M. E. \& Thomas, R. The taming of cyclobutadiene. Angew Chem. Int. Ed. 30, 1024-1027 (1991).

10. Warmuth, R. \& Marvel, M. A. 1,2,4,6-Cycloheptatetraene: room-temperature stabilization inside a hemicarcerand. Angew. Chem. Int. Ed. 39, 1117-1119 (2000).

11. Warmuth, R. o-Benzyne: strained alkyne or cumulene? - NMR characterization in a molecular container. Angew. Chem. Int. Ed. 36, 1347-1350 (1997).

12. Körner, S. K., Tucci, F. C., Rudkevich, D. M., Heinz, T. \& Rebek, Jr J. A self-assembled cylindrical capsule: new supramolecular phenomena through encapsulation. Chem. Eur. J. 6, 187-195 (2000).

13. Ziegler, M., Brumaghim, J. L. \& Raymond, K. N. Stabilization of a reactive cationic species by supramolecular encapsulation. Angew. Chem. Int. Ed. 39, 4119-4121 (2000).

14. Yoshizawa, M., Kusukawa, T., Fujita, M., Sakamoto, S. \& Yamaguchi, K. Cavity-directed synthesis of labile silanol oligomers within self-assembled coordination cages. J. Am. Chem. Soc. 123, 10454-10459 (2001).

15. Dong, V. M., Fiedler, D., Carl, B., Bergman, R. G. \& Raymond, K. N. Molecular recognition and stabilization of iminium ions in water. J. Am. Chem. Soc. 128, 14464-14465 (2006).

16. Iwasawa, T., Hooley, R. J. \& Rebek, Jr J. Stabilization of labile carbonyl addition intermediates by a synthetic receptor. Science 317, 493-496 (2007).

17. Mal, P., Breiner, B., Rissanen, K. \& Nitschke, J. R. White phosphorus is air-stable within a self-assembled tetrahedral capsule. Science 324, 1697-1699 (2009).

18. Nishimura, N. \& Kobayashi, K. Self-assembled boronic ester cavitand capsule as a photosensitizer and a guard nanocontainer against photochemical reactions of 2,6-diacetoxyanthracene. J. Org. Chem. 75, 6079-6085 (2010).

19. Horiuchi, S., Murase, T. \& Fujita, M. Noncovalent trapping and stabilization of dinuclear ruthenium complexes within a coordination cage. J. Am. Chem. Soc. 133, 12445-12447 (2011)

20. Fetzer, J. C. The Chemistry and Analysis of the Large Polycyclic Aromatic Hydrocarbons (John Wiley, 2000) 
21. Watson, M. D., Fechtenkoetter, A. \& Müllen, K. Big is beautiful-“aromaticity" revisited from the viewpoint of macromolecular and supramolecular benzene chemistry. Chem. Rev. 101, 1267-1300 (2001).

22. Sheppard, C. S. \& Kamath, V. R. Azo and Peroxide Free Radical Initiators (Technical Data, SPI, Lucidol, Pennwalt Corp., 1978).

23. Moad, G. \& Solomon, D. H. The Chemistry of Radical Polymerization (Elsevier, 2006).

24. Kishi, N., Li, Z., Yoza, K., Akita, M. \& Yoshizawa, M. An $M_{2} L_{4}$ molecular capsule with an anthracene shell: encapsulation of large guests up to $1 \mathrm{~nm}$. J. Am. Chem. Soc. 133, 11438-11441 (2011).

25. Kishi, N. et al. Wide-ranging host capability of a $\mathrm{Pd}(\mathrm{II})$-linked $\mathrm{M}_{2} \mathrm{~L}_{4}$ molecular capsule with an anthracene shell. Chem. Eur. J. 19, 6313-6320 (2013).

26. Yoshizawa, M. \& Klosterman, J. K. Molecular architectures of multi-anthracene assemblies. Chem. Soc. Rev. 43, 1885-1898 (2014).

27. Kita, Y. et al. Addition of malononitrile radicals to alkenes under mild conditions using 2,2'-azobis(2,4-dimethyl-4-methoxyvaleronitrile) (V-70) as an initiator. Tetrahedron Lett. 38, 3549-3552 (1997).

28. Sun, C.-k., Zhao, H.-m., Fang, D.-C. \& Li, Z.-h. Theoretical study on the thermal decomposition of azoisobutyronitrile. J. Mol. Struct. 679, 89-94 (2004).

29. Kleywegt, G. J. \& Jones, T. A. Detection, delineation, measurement and display of cavities in macromolecular structures. Acta. Cryst. D 50, 178-185 (1994).

30. Ajami, D. \& Rebek, Jr J. More chemistry in small spaces. Acc. Chem. Res. 46, 990-999 (2013)

31. Ko, Y. H., Kim, H., Kim, Y. \& Kim, K. U-shaped conformation of alkyl chains bound to a synthetic host. Angew. Chem. Int. Ed. 47, 4106-4109 (2008).

32. Choudhury, R., Barman, A., Prabhakar, R. \& Ramamurthy, V. Hydrocarbons depending on the chain length and head group adopt different conformations within a water-soluble nanocapsule: ${ }^{1} \mathrm{H}$ NMR and molecular dynamics studies. J. Phys. Chem. B 117, 398-407 (2013).

33. Kishi, N. et al. Facile catch and release of fullerenes using a photoresponsive molecular tube. J. Am. Chem. Soc. 135, 12976-12979 (2013).

\section{Acknowledgements}

This work was supported by the Japan Society for the Promotion of Science (JSPS) through a 'Funding Program for Next-Generation World-Leading Researchers' and by the Japanese Ministry of Education, Culture, Sports, Science and Technology (MEXT) through Grants-in-Aid for Scientific Research on Innovative Areas 'Soft Molecular Systems'. We also thank Dr Kenji Yoza (Bruker AXS) for supporting X-ray crystallographic analysis, Professor Daisuke Takeuchi and Dr Fumitaka Ishiwari (Tokyo Institute of Technology) for supporting GPC measurement, Dr Yu Fang (The University of Tokyo) for supporting VOIDOO calculation and Professor Jeremy K. Klosterman (Bowling Green State University) for helpful discussions.

\section{Author contributions}

M.Ya. and M.Yo. designed the work, carried out research, analysed data, and wrote the paper. Y.S. contributed to crystallographic analysis. M.A. was involved in the work discussion and M.Yo. is the principal investigator. All authors discussed the results and commented on the manuscript.

\section{Additional information}

Accession codes: CCDC-990095 and 990110 contain the supplementary crystallographic data for the structures reported in this article. These data can be obtained free of charge from The Cambridge Crystallographic Data Centre (CCDC) via www.ccdc.cam.ac.uk/ data_request/cif.

Supplementary Information accompanies this paper at http://www.nature.com/ naturecommunications

Competing financial interests: The authors declare no competing financial interests.

Reprints and permission information is available online at http://npg.nature.com/ reprintsandpermissions/

How to cite this article: Yamashina, M. et al. Safe storage of radical initiators within a polyaromatic nanocapsule. Nat. Commun. 5:4662 doi: 10.1038/ncomms5662 (2014). 